\title{
Joint expedition: linking gut inflammation to arthritis
}

\author{
P Jacques $^{1}$ and D Elewaut ${ }^{1}$
}

The tight connection between intestinal inflammation and arthritis in spondyloarthritis (SpA) has been studied extensively. Subclinical gut inflammation, which can be considered as a model for early Crohn's disease, was shown to be strongly associated with joint inflammation. Several early mucosal abnormalities were uncovered even in the absence of histological signs of inflammation, providing clues into the pathogenesis of SpA. Nevertheless, many questions remain unanswered. In this review, we highlight recent progress on this intimate relationship between gut and joint inflammation. Emerging evidence exists favoring a role for genes beyond human leukocyte antigen B27 in the genetic predisposition of SpA and intestinal inflammation. Furthermore, the role of these predisposing genes in modulating host-pathogen interaction at mucosal surfaces and the subsequent link between gut and joint inflammation are of utmost importance in understanding the pathogenesis of SpA.

\section{MUCOSAL INFLAMMATION AND CHRONIC SYNOVITIS: THE SCENERY}

Chronic inflammatory arthritis, a hallmark of a variety of inflammatory rheumatic diseases, and inflammatory bowel disease (IBD) are life-long conditions, often with onset in early adulthood, with an important morbidity and even mortality in our society. These diseases are not uncommon as approximately $2-3 \%$ suffers from chronic arthritis in our society. Interestingly, a coexistence of gut and joint inflammation was found to be prominent in spondyloarthritis ( $\mathrm{SpA})$, a cluster of interrelated rheumatologic diseases, characterized by a number of clinical and genetic features including peripheral arthritis (typical of lower limb joints) as well as inflammation of the axial skeleton (e.g., spine). Remarkably, these diseases may also affect other organs including skin (psoriasis) or the eye (anterior uveitis), indicating the systemic nature of these diseases. Various subtypes of SpA can be distinguished based upon the clinical features, but an important overlap may occur between either of them. They include ankylosing spondylitis (AS, characterized by prominent inflammation of the axial skeleton (spine, sacroiliac joints), although other joints may also be affected), infection-triggered reactive arthritis $(\operatorname{ReA})$, some forms of juvenile chronic arthritis, arthritis in association with IBD, and some forms of psoriatic arthritis. Intriguingly, approximately twothirds of patients suffering from SpA have microscopic signs of gut inflammation without clinical gastrointestinal symptoms. ${ }^{1-4}$
Furthermore, $6-13 \%$ of these patients eventually develop IBD, particularly Crohn's disease (CD). ${ }^{5-9}$ The occurrence of subclinical bowel inflammation was shown to appear in all known SpA subsets. Strikingly, a remarkable clinical relationship between gut and joint inflammation was revealed in prospective follow-up studies as gut inflammation occurred more frequently in SpA patients suffering from peripheral arthritis compared with patients without this condition. Similarly, remission of joint inflammation was associated with disappearance of gut inflammation. Conversely, persistence of peripheral arthritis was usually accompanied by persistence of bowel inflammation. ${ }^{6-8}$

An early indication for a possible relationship between inflammation of the mucosal immune system and peripheral arthritis was provided by the observation that peripheral joint inflammation may appear in genetically predisposed patients presenting with certain types of bacterial gut infections such as Salmonella typhimurium, Yersinia enterocolitica, Shigella, and Campylobacter jejuni. About $20 \%$ of the patients with this so-called ReA eventually evolve into AS, leading to severe disability. ${ }^{10}$ Two decades ago, it was shown that a high frequency of inflammatory gut lesions could be observed in patients from various subtypes of SpA presenting with inflammatory joint symptoms but lacking any clinical sign of gastrointestinal discomfort. ${ }^{1-4}$ This observation is particularly interesting because an anatomic linkage between these two organs is lacking. Inflammatory gut lesions

1Department of Rheumatology, Ghent University Hospital, Ghent, Belgium. Correspondence: D Elewaut (dirk.elewaut@ugent.be) 


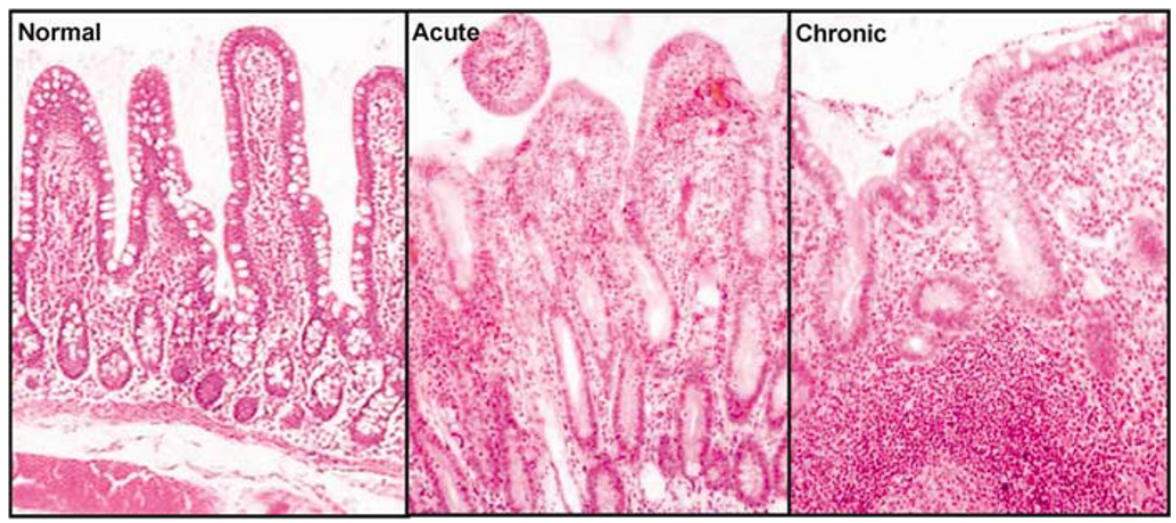

Figure 1 Subclinical bowel inflammation in spondyloarthritis. The left panel illustrates the normal histology of human ileum, with tall and lean villi. In the acute type of inflammation, architecture is well preserved, allthough an increase in inflammatory cells, particularly neutrophils, is obvious. In contrast, in the chronic type, villi are blunted and fused, and the edematous lamina propria is infiltrated by mononuclear cells with formation of granuloma.

are found in up to $90 \%$ of the patients, depending on the SpA subtype. ${ }^{11}$

In addition to shared clinical features, a number of common genetic predispositions were identified linking SpA and $\mathrm{CD}$ pathogenesis to immune-mediated inflammatory diseases. Hence, the strong genetic association of SpA with the human leukocyte antigen (HLA) B27 has extensively been documented ${ }^{12-15}$ and very recently, an association with interleukin (IL)-23 receptor polymorphisms in both CD and AS was reported. ${ }^{16-18}$

\section{FEATURES OF BOWEL INFLAMMATION IN SPONDYLOARTHRITIS}

The gut mucosal immune system has the important task to maintain tolerance against the continuous antigenic challenge, from birth to death, to food antigens and antigens of the abundant normal bacterial flora, and pathogens. To accomplish this, the intestine is equipped with a variety of components, each of them with well-defined roles in maintenance of homeostasis. They include the intestinal epithelial cells and intraepithelial lymphocytes, which in humans mostly use the $\alpha \beta$ T-cell receptor (TCR), with only $10 \% \gamma \mathrm{dTCRs}$, and the majority being CD8 ${ }^{+}{ }^{19}$ Practically all intraepithelial lymphocytes express the integrin $\alpha \mathrm{E} \beta 7$, which anchors intraepithelial lymphocytes in the epithelium by interactions with its ligand E-cadherin. Beneath the intestinal epithelial layer lies the lamina propria, which contains numerous lymphocytes, especially $\mathrm{T}$ cells, macrophages, dendritic cells, and plasma cells, all active players of the immune response of the gut. The intestinal epithelial cells have gathered much interest because of their unique position as initial sites of interaction between pathogens and the host. As such, they have the capacity to induce a coordinated and conserved response, characterized by the production of an array of cytokines and chemokines in response to invasion by pathogens. ${ }^{20,21}$ In between the crypts, the Peyer's patches (PPs) or lymphoid follicles are found, which are organized areas of lymphoid tissue, covered by follicle-associated epithelium. PPs contain a follicle center, surrounded by a mantle of small lymphocytes. The dome area around this center contains plasma cells, dendritic cells, macrophages, and B cells and in between the follicles, zones of $\mathrm{T}$ cells are found. The follicle-associated epithelium overlying the PP is different from the normal epithelium in that it has fewer goblet cells and many specialized $M$ cells, which are in very close contact with $\mathrm{B}$ and $\mathrm{T}$ lymphocytes in the PP and have a role in antigen entry into the follicle. ${ }^{22}$ Due to this highly organized structure, the gut is privileged to be a key player in diverse immunological processes. Mucosal alterations are one of the first signs of ongoing inflammation in SpA. Histologically, the inflammation can be divided in acute and chronic types (Figure 1). In the first type, mucosal architecture is well-preserved, mimicking an acute and selflimiting bacterial enterocolitis. In contrast, in the chronic type of gut inflammation, intestinal architecture is profoundly altered, with blunted and fused villi. The crypts are distorted and the lamina propria is edematous, and infiltrated by mononuclear cells, instead of the primarily neutrophilic dominance in acute inflammation. ${ }^{1}$

In this review, we will focus on events linking the gut to joint inflammation in SpA.

The discovery of microscopic signs of gut inflammation in SpA patients has set off a cascade of intriguing studies, providing evidence for an early link between the gut mucosal immune system and joint inflammation. Several early alterations are listed below.

Cadherins are cell-adhesion molecules that play diverse functions in cell-cell interactions. In mice, disruption of N-cadherin, which is involved in maintaining the integrity of the intestinal epithelial layer, results in the occurrence of IBD, mimicking CD. ${ }^{23}$ In humans, homotypic homophilic intercellular adhesion of intestinal epithelial cells is mediated by E-cadherin. In addition, E-cadherin also functions as a ligand for $\alpha \mathrm{E} \beta 7$ integrin, which is abundantly expressed on intraepithelial lymphocytes. An upregulation of E-cadherin and its associated catenins was demonstrated in IBD. ${ }^{24}$ In SpA patients with acute or chronic subclinical gut inflammation, expression of the proteins of the E-cadherin/catenin complex was found to be increased as well, especially at the sites of active inflammation. ${ }^{24,25}$ It is of interest to note that expression of the E-cadherin ligand, $\alpha E \beta 7$ 
integrin, was also found to be upregulated on mucosal T cells from colon of AS patients, without histological signs of inflammation by routine pathological evaluation using hematoxylin and eosin-stained tissue sections (hereafter referred to as "noninflamed"). ${ }^{26}$ Furthermore, the number of lymphoid follicles was found to be increased in the ileum as well as in the colon of SpA patients, in the absence of macroscopic or microscopic signs of gut inflammation, compared to healthy controls. ${ }^{27}$ Similarly, the fraction of lamina propria mononuclear cells expressing the adhesion molecules CD11a, CD11c, and VCAM-1 was also increased in non-inflamed gut mucosa from SpA patients. Macrophages, characterized by the expression of CD68, were also more numerous in the colon mucosa from SpA patients. ${ }^{27}$ In addition, in SpA patients, $M$ cells were found to be damaged in inflamed ileal mucosa. ${ }^{28}$

To extend these observations, gene array studies were conducted on intestinal biopsies from non-inflamed areas from a group of SpA patients and compared to non-inflamed specimens from $\mathrm{CD}$ patients and healthy controls. It was found that patients with SpA have an altered mucosal gene expression profile compared with healthy controls: ${ }^{29}$ a set of 95 genes that are differentially expressed in the colon of patients with $\mathrm{CD}$ and with SpA vs. controls was identified. This suggests that $\mathrm{SpA}$ patients with chronic subclinical gut inflammation tend to cluster with $\mathrm{CD}$ patients by means of expression of these genes. Two genes in this set had already been described in the context of CD: acyl-coenzyme A oxidase (ACOX1), the activity of which was reported to be reduced in $\mathrm{CD}$ patients, and glutathione peroxidase 2 , an enzyme that is exclusively expressed in the intestine and even overexpressed in the normal colonic tissue of patients with $\mathrm{CD}$ and in SpA patients with a history of chronic gut inflammation. ${ }^{30,31}$ These findings suggest that some of these genes could be important as early genetic markers for evolution to CD in SpA.

Although the precise role of these various perturbations in the intestinal mucosal immune system in the induction of arthritis remains to be determined, overall, these findings indicate that in $\mathrm{SpA}$, early alterations in the gut mucosal immune system may be observed, which may be involved in the onset of histological overt signs of inflammation.

\section{LINKING GUTTO JOINT INFLAMMATION}

Several trails attempting to link mucosal and joint inflammation have been followed during the past decades. One theory points at the possible role for gut bacteria in the origin of articular inflammation. The most popular theories, however, involve aberrant migration of intestinal lymphocytes or mononuclear cells, particularly macrophages. Although neither of these theories has been formally proven, experimental evidence has been gathered over the past years supporting either of these hypotheses. Only recently, data from an animal model with combined gut and joint inflammation provided the first strong evidence for a common pathophysiological mechanism. TNF receptor I (TNFRI) expression within the stromal compartment appeared a sufficient target for TNF in the development of both gut and joint inflammation. ${ }^{32}$
The gut mucosal immune system is the first barrier against invasion of pathogenic microorganisms. A dysfunctional interaction between the mucosal immune system and gut bacteria could result in an abnormal state of immunological tolerance toward flora by alterations in mucosal effector cells or by affecting regulatory cells. Changes in intestinal flora itself is yet another important contributing factor, for example, due to an abnormal number of microorganisms or by changes in the composition of the microbial flora.

In patients with ReA, the triggering infectious organisms have been extensively described and typically involve Gram-negative bacteria that have the capacity to invade host cells and survive intracellularly. ${ }^{33}$ It is believed that persistent infection or at least persistence of bacterial components within the body ultimately results in the occurrence of arthritis. Bacterial antigens from $S$. typhimurium and Y. enterocolitica were found in synovial fluid of inflamed joints of ReA patients. ${ }^{34,35}$ Both synovial fluid mononuclear and polymorphonuclear phagocytes stained positive for lipopolysaccharide components. However, no intact bacteria could be detected, and extensive bacterial cultures remained negative. Using the more sensitive PCR techniques, bacterial DNA from several ReA-associated bacteria could be detected in synovial fluid; ${ }^{36,37}$ however, rRNA from many other microorganisms, such as Escherichia coli and other commensal flora could be distinguished as well. ${ }^{38}$ This may be compatible with an intestinal origin of these bacterial components.

The most compelling evidence for the pathogenic role of bacteria in the pathogenesis of gut and joint inflammation in $\mathrm{SpA}$ is derived from animal models. In the $H L A-B 27 /$ human $\beta 2$-microglobulin transgenic rat model, a high copy number of the transgene (55-150 copies) is necessary to induce an SpAlike disease in genetically predisposed strains (Lewis or Fisher), characterized by the development of sacroiliitis, spondylitis, and peripheral arthritis. Furthermore, extra-articular manifestations including psoriasiform skin and nail lesions and interestingly, bowel inflammation (enterocolitis), also occur in this model. Remarkably, these rats remain free from gut and joint disease when kept in germ-free conditions, reflecting the interplay between predisposing genes and bacteria. ${ }^{39}$

The second set of theories focuses on aberrant trafficking of lymphocytes or mononuclear cells.

Naive lymphocytes, once matured in bone marrow and thymus, continuously recirculate in between different lymphoid organs until they encounter a specific antigen. Following antigen encounter in the secondary lymphoid organs, including mesenteric lymph nodes or PPs, lymphocytes proliferate and differentiate, hereby expressing a certain set of adhesion molecules on their membrane. Mature lymphocytes then home to sites that are similar to those where the original antigen encounter took place (reviewed in ref. 40). Lymphocyte homing is mediated by an array of adhesion molecules, such as integrins and selectins, and by chemokine receptors. The homing of intestinal lymphocytes is regulated by a limited set of adhesion molecules. The $\beta 7$ integrin subfamily, the expression of which is limited to leukocytes, includes two important members playing a pivotal role in homing to the intestine: $\alpha 4 \beta 7$ and $\alpha \mathrm{E} \beta 7 .{ }^{40}$ The $\alpha 4 \beta 7$ 
integrin serves as a ligand for MadCAM-1, the mucosal vascular addressin that is selectively expressed by mucosal endothelial cells, whereas $\alpha \mathrm{E} \beta 7$ integrin is constitutively expressed by intraepithelial $\mathrm{T}$ cells in the intestinal mucosa and binds to E-cadherin, expressed on gut epithelial cells. ${ }^{41}$ Over the past decade, several groups have addressed this hypothesis, by distinct approaches. First, Salmi et al. ${ }^{42}$ demonstrated that mucosal immunoblasts, isolated from inflamed bowel from patients suffering from IBD, are able to bind in vitro to inflamed synovial vessels rather than to control tissues such as peripheral lymph nodes, suggesting that altered trafficking of leukocytes may contribute to the pathogenesis of arthritis in IBD. ${ }^{43} \mathrm{~A}$ distinct repertoire of adhesion molecules is utilized for this purpose, involving vascular adhesion protein-1. It should be noted that in these studies, no inflamed tissues such as skin from psoriasis patients were evaluated in the in vitro adhesion assays of mucosal leukocytes. This is particularly relevant as later on it was shown that vascular adhesion protein-1 is upregulated in a variety of inflammatory conditions, such as inflamed skin. ${ }^{44,45}$ Consistent with the previous data, it was shown that in early SpA patients, activated $\mathrm{T}$ cells carrying the $\alpha 4 \beta 7$ and $\alpha \mathrm{E} \beta 7$ integrins were enriched in inflamed synovial tissue, ${ }^{46}$ suggesting a mucosal origin of these $\mathrm{T}$ cells. However, as the expression of $\beta 7$ integrins may also be modulated during $\mathrm{T}$-cell activation or by certain cytokines, including transforming growth factor- $\beta,{ }^{47,48}$ the evidence linking this altered expression of $\beta 7$ integrin expression on synovial T cells to gut inflammation is only circumstantial. If intestinal T cells in SpA patients migrate to the joints, then identical clonally expanded $\mathrm{T}$ cells must be present in both intestine and joint. T-cell clones, specific for enterobacterial antigens, have been derived from the synovial fluid or membrane of patients with ReA, a condition in which a full-blown SpA can develop following a bacterial gut infection. ${ }^{49,50}$ May et al. ${ }^{51}$ were able to demonstrate for the first time the existence of identical clones in a patient with enterogenic SpA. However, it is important to highlight that only a few expanded T-cell clones were shared by both compartments, so this phenomenon cannot be simply explained by intestinal activation. In accordance with this theory is the observation that $H L A-B 27$-restricted CD8+ T-cell clones could be isolated from the synovial fluid of patients with AS or ReA. ${ }^{52}$ The identified T-cell clones specifically recognized the enterobacteria Y. enterocolitica and S. Typhimurium, both associated with ReA. HLA-B27 could contribute to disease pathogenesis by its potential capacity to present arthritogenic peptides, autologous or bacterial, to $\mathrm{CD} 8+\mathrm{T}$ cells, although alternate theories on the role of $H L A-B 27$ in SpA pathogenesis have been formulated.

Nevertheless, the aberrant migration of lymphocytes does not explain the presence of bacterial components in synovial fluid or tissue from SpA patients.

As macrophages are primary cellular targets for the intracellular pathogens associated with $\mathrm{SpA}$, it was postulated that trafficking of mononuclear cells from gut to joint would be a critical factor in the relation between gut and joint inflammation. Thus, macrophages could contribute to disease pathogenesis by the uptake of bacterial components in the intestine, with subsequent presentation to T cells and migration to the target joint (Figure 2). Consistent herewith, a particular subset of macrophages, expressing the scavenger receptor CD163, was found to be enriched in colon of patients with SpA and CD, even in non-inflamed regions. ${ }^{53}$ This specific subset is also selectively increased in SpA synovium, ${ }^{54}$ and global disease activity correlated well with the number of $\mathrm{CD}_{163}{ }^{+}$macrophages and polymorphonuclear cells in the synovium. ${ }^{55}$ In addition, Salmi et al. ${ }^{56}$ demonstrated that macrophages isolated from the lamina propria from human intestine can bind in vitro to vessels from inflamed human synovial tissue. Unlike the binding of lymphocytes, macrophage adhesion to synovial endothelium was reported to be almost entirely dependent upon P-selectin.

Recently, an alternative explanation for the observed relation between gut and joint inflammation in SpA was revealed in the TNF $\triangle A R E$ mouse model. By deletion of the AU-rich elements in the regulatory sequences of the murine TNF genome, a chronic and dysregulated TNF production occurred leading to an inflammatory disease characterized by the simultaneous occurrence of a Crohn-like IBD and an articular disease. ${ }^{57}$ Recently, it was shown that this articular disease involves both peripheral synovitis and sacroiliitis, thereby covering many features of the SpA concept. ${ }^{32}$ Signaling through TNFRI was previously shown to be mandatory for the development of both the bowel inflammation and the arthritis, ${ }^{57}$ but the cellular targets of TNF remained poorly defined. Recently, however, Armaka et al. ${ }^{32}$ demonstrated using Cre/loxP-mediated TNFR1 expression in mesenchymal cells that TNFRI expression within the stromal compartment provided a sufficient target for TNF in the development of both gut and joint inflammation. Therefore, in the presence of chronic TNF overexposure, signaling through TNFRI in synovial fibroblasts and intestinal myofibroblasts appears to be sufficient to develop combined gut and joint pathologies, a hallmark of SpA.

\section{PREDISPOSING GENES IN COMBINED GUT AND JOINT DISEASE}

Which patients eventually develop both gut and joint inflammation seems partially determined by their genetic predisposition.

Recently, the $I L-23$ receptor $(I L-23 R$ ) gene on chromosome 1 p31 was identified as a susceptibility determinant for CD. An uncommon coding variant (rs11209026, Arg381Gln), in which arginine is substituted by glutamine, has been shown to confer strong protection against CD. ${ }^{17}$ IL-23 was originally reported to be a proinflammatory cytokine, as overexpression of its p19 subunit caused systemic inflammation in transgenic animals. ${ }^{58} \mathrm{In}$ a spontaneous model of enterocolitis, the IL-10-deficient mice, IL-23 appeared to be important as well. ${ }^{59}$ Double knockout mice ( $\left.I L 10^{-/-} \times p 19^{-/-}\right)$remained free from intestinal inflammation for up to 12 months of age, whereas controls exhibited severe enterocolitis. In view of the intimate relationship between intestine and joints in SpA, three independent research groups investigated the possible association of $I L-23 R$ variants in different cohorts of patients with AS and controls. They all reported the same association with this polymorphism. ${ }^{16,18,60}$ 


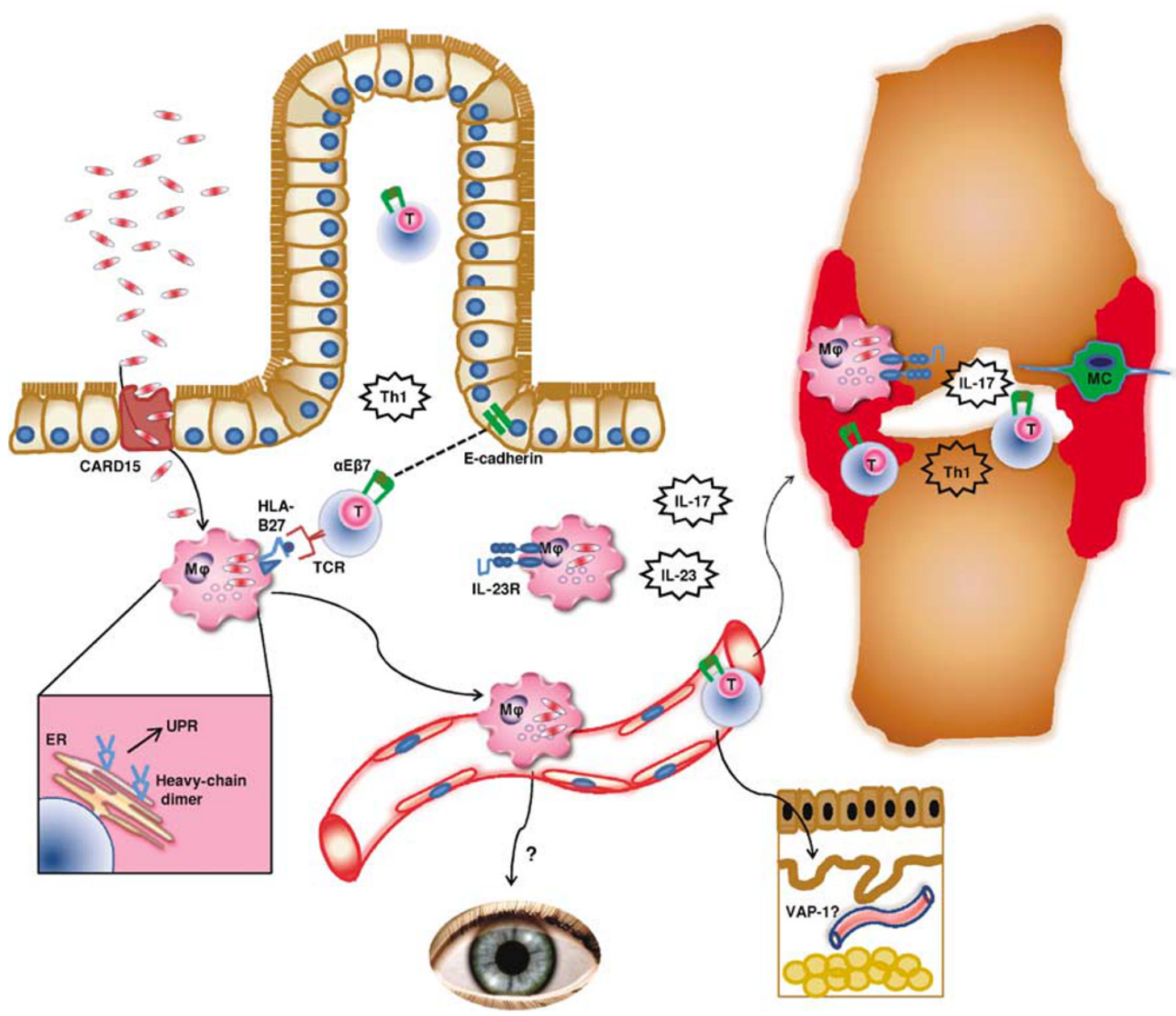

Figure $2 \mathrm{~A}$ model linking gut to joint inflammation in spondyloarthritis. (1) Bacteria attach to and invade the intestinal epithelium and the lamina propria. HLA-B27 or CARD15 polymorphisms can result in altered recognition and handling of bacterial antigens. (2) Invading bacteria infect, or are taken up by, macrophages (M) in the lamina propria and survive intracellularly. (3) HLA-B27 can present bacterial or autologous antigens to T cells (T). Furthermore, the heavy chain easily misfolds, leading to an unfolded protein response (UPR) and stress. (4) Bacterial infection induces Th1 and Th17 responses, and IL-23R susceptibility variants, expressed on macrophages and other antigen-presenting cell types, may modulate the Th17 response. (5) More T cells and other immune mediators are recruited, releasing proinflammatory cytokines. (6) Activated T cells and macrophages carrying bacterial components migrate via blood vessels to the target joint or eventually to other sites such as skin and eye. (7) In the target joint, gut-derived macrophages and T cells recruit other immune cells and result in the activation of mesenchymal cells (MCs), which further enhance and sustain inflammation. HLA-B27, human leukocyte antigen B27; IL-23R, IL-23 receptor.

However, the putative association of the $I L-23 R$ variants with subclinical bowel inflammation in SpA remains to be investigated.

The relationship between $H L A-B 27$ and AS has been recognized for more than 30 years and appears to be one of the strongest associations between an $\mathrm{MHC}$ gene and disease known to date. Indeed, 75-95\% of AS patients are HLA-B27positive. ${ }^{12-14}$ In contrast, the frequency of this molecule in the general IBD population was found to be comparable with the healthy control population. However, in patients with IBD who also had spondylitis or sacroiliitis, the prevalence of $H L A-B 27$ was higher (25-78\%), suggesting that $H L A-B 27$ is a risk factor for the development of AS in these patients. ${ }^{13,15,61}$ The HLA-B27 molecule could be implicated in disease pathogenesis in several ways. One theory points at the structural characteristics of the HLA-B27 molecule, which is composed of a trimer of heavy chain, $\beta 2$-microglobulin and short peptide. Normally, the tertiary structure of major histocompatibility complex class I molecules is easily achieved by the aid of chaperones. However, the folding rate of the HLA-B27 heavy chain is unusually slow and therefore it tends to misfold, to accumulate in the endoplasmatic reticulum, thereby forming disulphide-linked homodimers. ${ }^{62}$ Accumulation of unfolded heavy chain may generate stress, a so-called unfolded protein response, which can induce profound changes in the cellular metabolism, such as inhibition of general translation but transcriptional upregulation of molecular chaperone genes. Prolonged or acute endoplasmatic reticulum stress results in cell death. ${ }^{63} \mathrm{~A}$ recent study in HLA-B27 transgenic rats confirmed for the first time the relation between a high copy number of HLA-B27, misfolding of its heavy chain, and accumulation in the endoplasmatic reticulum, followed by unfolded protein response and arthritis. ${ }^{64,65}$ Moreover, 
additional human $\beta 2$-microglobulin was able to reduce the unfolding of B27 and subsequent unfolded protein response in HLA-B27 transgenic rats. ${ }^{66}$ Surprisingly, these rats more frequently displayed a more severe arthropathy whereas, in contrast, colitis was completely abolished. The clinical and histopathological features of this arthropathy more adequately resembled SpA than the original B27 transgenic rat model as axial involvement was found to be more prevalent. Thus, it appears that in this model, gut inflammation seems not mandatory for the development of articular disease.

These observations fail to provide an explanation for the development of SpA in HLA-B27-negative subjects and in addition, in human SpA patients, gut inflammation was previously found to occur more frequently in $H L A-B 27$-negative SpA patients. ${ }^{67}$ Hence, the contribution of $H L A-B 27$ into the overall genetic predisposition is only estimated to be around $16 \%$, as concluded from twin studies. ${ }^{68}$ These questions have sparked the search for additional predisposing genes.

A gene that has gathered much interest is CARD15, coding for a protein that functions as an intracellular pattern-recognition receptor through its leucin-like repeats and by consequence can act as an intracellular receptor for bacterial pattern molecules in mammalian cells. Binding results in NF- $\mathrm{KB}$ activation and in apoptosis through engagement of its two $\mathrm{N}$-terminal caspase-recruitment domains. Interest in CARD15 has arisen from the well-documented correlation between CARD15 (NOD2) gene polymorphisms and an increased susceptibility for CD. ${ }^{69-71}$

Although the precise role of CARD15 polymorphisms in the pathogenesis of $\mathrm{CD}$ is not yet fully understood, evidence has been provided that CARD15 is a negative regulator of the TLR2-mediated Th1 response. In the presence of CARD15 variants, no inhibition of TLR2-mediated signaling occurs resulting in a prominent bias toward a Th1-mediated response. ${ }^{72}$ Recently, CARD15 in dendritic cells has also been linked to the generation of Th17 cells. ${ }^{73}$

CARD15 is expressed by monocytes, dendritic cells, Paneth cells, and intestinal epithelial cells, ${ }^{74-78}$ and its expression can be regulated by interaction with bacterial components, even from non-pathogenic commensal E. coli. ${ }^{79}$ Three independent single-nucleotide polymorphisms (one frameshift mutation and two missense mutations) of CARD15 are associated with $\mathrm{CD}$ in $30-46 \%$ of patients. ${ }^{69-71}$ These variants increase the risk for CD threefold for heterozygous and 38- and 44-fold, respectively, for homozygous and compound heterozygous individuals. These data suggest that the impairment of normal host-microbial interactions may contribute to the pathogenesis of CD.

In SpA patients, the overall prevalence of CARD15 polymorphisms was not altered compared with healthy controls. ${ }^{80-82}$ However, CARD15 polymorphisms in patients with SpA were associated with a higher risk of evolution to chronic gut inflammation. ${ }^{81}$

Thus, although a clear association between CARD15 polymorphisms and arthritis is absent, there is definitely an association with the development of gut inflammation in SpA patients.

Taken together, a general dysbiosis in combination with CARD15 polymorphisms could profoundly alter host- bacteria interactions in $\mathrm{CD}$ and SpA patients, resulting in enhanced intracellular survival of pathogenic organisms and subsequent aberrant immune activation.

It is unclear whether other recently identified predisposing genes in AS such as ARTS-1 also contribute to extra-articular manifestations. ${ }^{16}$ ARTS- 1 is involved in trimming of peptides to the optimal length required for presentation by major histocompatibility complex class I molecules. Furthermore, it contributes to shedding of cell-surface receptors of pro-inflammatory cytokines like TNF (TNFRI), IL-1, and IL- $6 .{ }^{83-85}$ Both functions provide an explanation for the association with AS: loss-of-function polymorphisms of ARTS-1 may result in presentation of abnormal peptides. Second, the normal downregulation of proinflammatory cytokine signaling may be compromised, resulting in enhanced pro-inflammatory effects.

Overall, these findings highlight the complex genetic background underlying the pathogenesis of SpA and in particular, the relation between gut and joint inflammation. Nevertheless, the data point to an important role of predisposing genes on the immune system by affecting innate and adaptive immunity.

\section{REMAINING QUESTIONS}

Although several breakthroughs have been realized in the genetic predisposition of AS over the past years, several issues, however, remain unclear. In particular, it remains to be determined whether similar predisposing genes contribute to articular vs. extra-articular (gut, eye, and skin) involvement in this disease. Likewise, genetic factors contributing to chronicity of disease also need to be identified. The role of novel identified associations, such as $I L-23 R$, will have to be explored, especially as many of the associated single-nucleotide polymorphisms were associated with a protection against disease. In addition, the potential interaction between HLA-B27, IL-23R polymorphisms, and perhaps ARTS-1, in the pathogenesis of the gut-joint axis will require additional efforts, from geneticists as well as immunologists. In this perspective, it is important to highlight that all of these identified genes largely affect adaptive immune responses. However, many gaps still exist in our understanding of the interplay between the host and pathogens, one of the initiating events in the disease. Hence, most of these studies have been focussing on HLA-B27 using transfectant cell lines, resulting in a variety of different hypotheses. Nevertheless, no clear consensus has been achieved yet on the role of HLA-B27 in modulating host-pathogen interactions. For the recently identified predisposing genes, a role in modulating these triggering events remains elusive. Thus, more than two decades after the initial discovery of subclinical bowel inflammation in $\mathrm{SpA}$, the underlying mechanism(s) remain in part a mystery and incite us to pursue further investigations.

\section{ACKNOWLEDGMENTS}

This work was supported by the Research Foundation Flanders, and the Research Council of Ghent University.

\section{DISCLOSURE}

The authors have no financial conflict of interest.

(C) 2008 Society for Mucosal Immunology 


\section{REFERENCES}

1. Cuvelier, C. et al. Histopathology of intestinal inflammation related to reactive arthritis. Gut 28, 394-401 (1987).

2. De Vos, M. et al. lleocolonoscopy in seronegative spondylarthropathy. Gastroenterology 96, 339-344 (1989).

3. Mielants, H., Veys, E.M., Cuvelier, C., De Vos, M. \& Botelberghe, L. HLA$\mathrm{B} 27$ related arthritis and bowel inflammation. Part 2. lleocolonoscopy and bowel histology in patients with HLA-B27 related arthritis. J. Rheumatol. 12, 294-298 (1985)

4. Mielants, H., Veys, E.M., Joos, R., Cuvelier, C. \& De Vos, M. Repeat ileocolonoscopy in reactive arthritis. J. Rheumatol. 14, 456-458 (1987).

5. Mielants, H., Veys, E.M., Cuvelier, C. \& de Vos, M. lleocolonoscopic findings in seronegative spondylarthropathies. Br. J. Rheumatol. 27 (Suppl 2), 95-105 (1988).

6. Mielants, H. et al. The evolution of spondyloarthropathies in relation to gut histology. II. Histological aspects. J. Rheumatol. 22, 2273-2278 (1995).

7. Mielants, $\mathrm{H}$. et al. The evolution of spondyloarthropathies in relation to gut histology. III. Relation between gut and joint. J. Rheumatol. 22, 2279-2284 (1995)

8. Mielants, H. et al. The evolution of spondyloarthropathies in relation to gut histology. I. Clinical aspects. J. Rheumatol. 22, 2266-2272 (1995).

9. De Vos, M., Mielants, H., Cuvelier, C., Elewaut, A. \& Veys, E. Long-term evolution of gut inflammation in patients with spondyloarthropathy. Gastroenterology 110, 1696-1703 (1996).

10. Leirisalo-Repo, M. Prognosis, course of disease, and treatment of the spondyloarthropathies. Rheum. Dis. Clin. North Am. 24, 737-751 viii (1998).

11. Mielants, H., Veys, E.M., Cuvelier, C. \& De Vos, M. Subclinical involvement of the gut in undifferentiated spondylarthropathies. Clin. Exp. Rheumatol. 7, 499-504 (1989).

12. Brewerton, D.A. et al. Ankylosing spondylitis and HL-A 27. Lancet 1, 904-907 (1973).

13. Palm, O., Moum, B., Ongre, A. \& Gran, J.T. Prevalence of ankylosing spondylitis and other spondyloarthropathies among patients with inflammatory bowel disease: a population study (the IBSEN study). J. Rheumatol. 29, 511-515 (2002).

14. Purrmann, J. et al. HLA antigens in ankylosing spondylitis associated with Crohn's disease. Increased frequency of the HLA phenotype B27,B44. J. Rheumatol. 15, 1658-1661 (1988).

15. Steer, S. et al. Low back pain, sacroiliitis, and the relationship with HLA-B27 in Crohn's disease. J. Rheumatol. 30, 518-522 (2003).

16. Burton, P.R. et al. Association scan of 14,500 nonsynonymous SNPs in four diseases identifies autoimmunity variants. Nat. Genet. 39, 1329-1337 (2007).

17. Duerr, R.H. et al. A genome-wide association study identifies IL23R as an inflammatory bowel disease gene. Science 314, 1461-1463 (2006).

18. Rahman, P. et al. Association of interleukin-23 receptor variants with ankylosing spondylitis. Arthritis Rheum. 58, 1020-1025 (2008).

19. Spencer, J., Isaacson, P.G., Diss, T.C. \& MacDonald, T.T. Expression of disulfide-linked and non-disulfide-linked forms of the T cell receptor gamma/delta heterodimer in human intestinal intraepithelial lymphocytes. Eur. J. Immunol. 19, 1335-1338 (1989).

20. Elewaut, D. et al. NF-kappa B is a central regulator of the intestinal epithelial cell innate immune response induced by infection with enteroinvasive bacteria. J. Immunol. 163, 1457-1466 (1999).

21. Jung, H.C. et al. A distinct array of proinflammatory cytokines is expressed in human colon epithelial cells in response to bacterial invasion J. Clin. Invest. 95, 55-65 (1995).

22. MacDonald, T.T. The mucosal immune system. Parasite Immunol. 25, 235-246 (2003).

23. Hermiston, M.L. \& Gordon, J.I. Inflammatory bowel disease and adenomas in mice expressing a dominant negative $\mathrm{N}$-cadherin. Science 270, 1203-1207 (1995).

24. Demetter, P. et al. Focal up-regulation of E-cadherin-catenin complex in inflamed bowel mucosa but reduced expression in ulcer-associated cell lineage. Am. J. Clin. Pathol. 114, 364-370 (2000).

25. Demetter, P. et al. Subclinical gut inflammation in spondyloarthropathy patients is associated with upregulation of the E-cadherin/catenin complex. Ann. Rheum. Dis. 59, 211-216 (2000).

26. Van Damme, N. et al. Gut mucosal T cell lines from ankylosing spondylitis patients are enriched with alphaEbeta7 integrin. Clin. Exp. Rheumatol. 19, 681-687 (2001)
27. Demetter, P. et al. Increase in lymphoid follicles and leukocyte adhesion molecules emphasizes a role for the gut in spondyloarthropathy pathogenesis. J. Pathol. 198, 517-522 (2002).

28. Cuvelier, C.A. et al. M-cells are damaged and increased in number in inflamed human ileal mucosa. Histopathology 24, 417-426 (1994).

29. Laukens, D. et al. Altered gut transcriptome in spondyloarthropathy. Ann. Rheum. Dis. 65, 1293-1300 (2006).

30. Aimone-Gastin, I. et al. Studies on peroxisomes of colonic mucosa in Crohn's disease. Dig. Dis. Sci. 39, 2177-2185 (1994).

31. Tuzun, A. et al. Oxidative stress and antioxidant capacity in patients with inflammatory bowel disease. Clin. Biochem. 35, 569-572 (2002).

32. Armaka, M. et al. Mesenchymal cell targeting by TNF as a common pathogenic principle in chronic inflammatory joint and intestinal diseases. J. Exp. Med. 205, 331-337 (2008).

33. Granfors, K. Do bacterial antigens cause reactive arthritis? Rheum. Dis. Clin. North Am. 18, 37-48 (1992).

34. Granfors, K. et al. Yersinia antigens in synovial-fluid cells from patients with reactive arthritis. N Engl. J. Med. 320, 216-221 (1989).

35. Granfors, K. et al. Salmonella lipopolysaccharide in synovial cells from patients with reactive arthritis. Lancet 335, 685-688 (1990).

36. Taylor-Robinson, D., Gilroy, C.B., Thomas, B.J. \& Keat, A.C. Detection of Chlamydia trachomatis DNA in joints of reactive arthritis patients by polymerase chain reaction. Lancet 340, 81-82 (1992).

37. Gaston, J.S., Cox, C. \& Granfors, K. Clinical and experimental evidence for persistent Yersinia infection in reactive arthritis. Arthritis Rheum. 42, 2239-2242 (1999)

38. Cox, C.J., Kempsell, K.E. \& Gaston, J.S. Investigation of infectious agents associated with arthritis by reverse transcription PCR of bacterial rRNA. Arthritis Res. Ther. 5, R1-R8 (2003).

39. Taurog, J.D. et al. The germfree state prevents development of gut and joint inflammatory disease in HLA-B27 transgenic rats. J. Exp. Med. 180, 2359-2364 (1994)

40. Johansson-Lindbom, B. \& Agace, W.W. Generation of gut-homing T cells and their localization to the small intestinal mucosa. Immunol. Rev. 215, 226-242 (2007).

41. Cepek, K.L. et al. Adhesion between epithelial cells and T lymphocytes mediated by E-cadherin and the alpha E beta 7 integrin. Nature 372 , 190-193 (1994).

42. Salmi, M., Andrew, D.P., Butcher, E.C. \& Jalkanen, S. Dual binding capacity of mucosal immunoblasts to mucosal and synovial endothelium in humans: dissection of the molecular mechanisms. J. Exp. Med. 181, 137-149 (1995).

43. Salmi, M. \& Jalkanen, S. Human leukocyte subpopulations from inflamed gut bind to joint vasculature using distinct sets of adhesion molecules. J. Immunol. 166, 4650-4657 (2001).

44. Arvilommi, A.M., Salmi, M., Kalimo, K. \& Jalkanen, S. Lymphocyte binding to vascular endothelium in inflamed skin revisited: a central role for vascular adhesion protein-1 (VAP-1). Eur. J. Immunol. 26, 825-833 (1996).

45. Salmi, M., Kalimo, K. \& Jalkanen, S. Induction and function of vascular adhesion protein-1 at sites of inflammation. J. Exp. Med. 178, 2255-2260 (1993).

46. Elewaut, D. et al. Enrichment of T cells carrying beta7 integrins in inflamed synovial tissue from patients with early spondyloarthropathy, compared to rheumatoid arthritis. J. Rheumatol. 25, 1932-1937 (1998).

47. Austrup, F., Rebstock, S., Kilshaw, P.J. \& Hamann, A. Transforming growth factor-beta 1-induced expression of the mucosa-related integrin alpha E on lymphocytes is not associated with mucosa-specific homing Eur. J. Immunol. 25, 1487-1491 (1995).

48. Brew, R., West, D.C., Burthem, J. \& Christmas, S.E. Expression of the human mucosal lymphocyte antigen, $\mathrm{HML}-1$, by $\mathrm{T}$ cells activated with mitogen or specific antigen in vitro. Scand. J. Immunol. 41, 553-562 (1995).

49. Hermann, E., Fleischer, B., Mayet, W.J., Poralla, T. \& Meyer zum Buschenfelde, K.H. Response of synovial fluid T cell clones to Yersinia enterocolitica antigens in patients with reactive Yersinia arthritis. Clin. Exp. Immunol. 75, 365-370 (1989).

50. Lahesmaa, R. et al. Yersinia enterocolitica activates a Thelper type 1-like T cell subset in reactive arthritis. J. Immunol. 148, 3079-3085 (1992).

51. May, E. et al. Identical T-cell expansions in the colon mucosa and the synovium of a patient with enterogenic spondyloarthropathy. Gastroenterology 119, 1745-1755 (2000). 
52. Hermann, E., Yu, D.T., Meyer zum Buschenfelde, K.H. \& Fleischer, B. HLA-B27-restricted CD8 T cells derived from synovial fluids of patients with reactive arthritis and ankylosing spondylitis. Lancet 342, 646-650 (1993).

53. Demetter, P. et al. Colon mucosa of patients both with spondyloarthritis and Crohn's disease is enriched with macrophages expressing the scavenger receptor CD163. Ann. Rheum. Dis. 64, 321-324 (2005).

54. Baeten, D. et al. Macrophages expressing the scavenger receptor CD163: a link between immune alterations of the gut and synovial inflammation in spondyloarthropathy. J. Pathol. 196, 343-350 (2002).

55. Baeten, D. et al. Infiltration of the synovial membrane with macrophage subsets and polymorphonuclear cells reflects global disease activity in spondyloarthropathy. Arthritis Res. Ther. 7, R359-R369 (2005).

56. Salmi, M., Rajala, P. \& Jalkanen, S. Homing of mucosal leukocytes to joints. Distinct endothelial ligands in synovium mediate leukocyte-subtype specific adhesion. J. Clin. Invest. 99, 2165-2172 (1997).

57. Kontoyiannis, D., Pasparakis, M., Pizarro, T.T., Cominelli, F. \& Kollias, G. Impaired on/off regulation of TNF biosynthesis in mice lacking TNF AU-rich elements: implications for joint and gut-associated immunopathologies. Immunity 10, 387-398 (1999).

58. Wiekowski, M.T. et al. Ubiquitous transgenic expression of the IL-23 subunit p19 induces multiorgan inflammation, runting, infertility, and premature death. J. Immunol. 166, 7563-7570 (2001).

59. Yen, D. et al. IL-23 is essential for T cell-mediated colitis and promotes inflammation via IL-17 and IL-6. J. Clin. Invest. 116, 1310-1316 (2006).

60. Reveille JD, Z.X. \& Bradbury, L.A. IL-23R is a major determinant of ankylosing spondylitis risk-the TASC study. Ann. Rheum. Dis. 66, 112 (2007).

61. de Vlam, K. et al. Spondyloarthropathy is underestimated in inflammatory bowel disease: prevalence and HLA association. J. Rheumatol. 27, 2860-2865 (2000).

62. Mear, J.P. et al. Misfolding of HLA-B27 as a result of its B pocket suggests a novel mechanism for its role in susceptibility to spondyloarthropathies. J. Immunol. 163, 6665-6670 (1999).

63. Schroder, M. \& Kaufman, R.J. ER stress and the unfolded protein response. Mutat. Res. 569, 29-63 (2005).

64. Turner, M.J., Delay, M.L., Bai, S., Klenk, E. \& Colbert, R.A. HLA-B27 upregulation causes accumulation of misfolded heavy chains and correlates with the magnitude of the unfolded protein response in transgenic rats: implications for the pathogenesis of spondylarthritis-like disease. Arthritis Rheum. 56, 215-223 (2007).

65. Turner, M.J. et al. HLA-B27 misfolding in transgenic rats is associated with activation of the unfolded protein response. J. Immunol. 175, 2438-2448 (2005).

66. Tran, T.M. et al. Additional human beta2-microglobulin curbs HLA-B27 misfolding and promotes arthritis and spondylitis without colitis in male HLA-B27-transgenic rats. Arthritis Rheum. 54, 1317-1327 (2006).

67. Mielants, H., Veys, E.M., Goemaere, S., Cuvelier, C. \& De Vos, M. A prospective study of patients with spondyloarthropathy with special reference to HLA-B27 and to gut histology. J. Rheumatol. 20, 1353-1358 (1993).

68. Sims, A.M., Wordsworth, B.P. \& Brown, M.A. Genetic susceptibility to ankylosing spondylitis. Curr. Mol. Med. 4, 13-20 (2004).

69. Hampe, J. et al. Association between insertion mutation in NOD2 gene and Crohn's disease in German and British populations. Lancet 357, 1925-1928 (2001).

70. Hugot, J.P. et al. Association of NOD2 leucine-rich repeat variants with susceptibility to Crohn's disease. Nature 411, 599-603 (2001).

71. Ogura, Y. et al. A frameshift mutation in NOD2 associated with susceptibility to Crohn's disease. Nature 411, 603-606 (2001).

72. Watanabe, T., Kitani, A., Murray, P.J. \& Strober, W. NOD2 is a negative regulator of Toll-like receptor 2-mediated Thelper type 1 responses. Nat. Immunol. 5, 800-808 (2004).

73. van Beelen, A.J. et al. Stimulation of the intracellular bacterial sensor NOD2 programs dendritic cells to promote interleukin-17 production in human memory T cells. Immunity 27, 660-669 (2007).

74. Hisamatsu, T. et al. CARD15/NOD2 functions as an antibacterial factor in human intestinal epithelial cells. Gastroenterology 124, 993-1000 (2003).

75. Lala, S. et al. Crohn's disease and the NOD2 gene: a role for paneth cells. Gastroenterology 125, 47-57 (2003).

76. Ogura, Y. et al. Nod2, a Nod1/Apaf-1 family member that is restricted to monocytes and activates NF-kappaB. J. Biol. Chem. 276, 4812-4818 (2001).

77. Ogura, Y. et al. Expression of NOD2 in Paneth cells: a possible link to Crohn's ileitis. Gut 52, 1591-1597 (2003).

78. Rosenstiel, P. et al. TNF-alpha and IFN-gamma regulate the expression of the NOD2 (CARD15) gene in human intestinal epithelial cells. Gastroenterology 124, 1001-1009 (2003).

79. Begue, B. et al. Microbial induction of CARD15 expression in intestinal epithelial cells via toll-like receptor 5 triggers an antibacterial response loop. J. Cell Physiol. 209, 241-252 (2006).

80. Crane, A.M. et al. Role of NOD2 variants in spondylarthritis. Arthritis Rheum. 46, 1629-1633 (2002).

81. Laukens, D. et al. CARD15 gene polymorphisms in patients with spondyloarthropathies identify a specific phenotype previously related to Crohn's disease. Ann. Rheum. Dis. 64, 930-935 (2005).

82. Miceli-Richard, C. et al. CARD15/NOD2 analyses in spondylarthropathy. Arthritis Rheum. 46, 1405-1406 (2002).

83. Cui, X. et al. Identification of ARTS-1 as a novel TNFR1-binding protein that promotes TNFR1 ectodomain shedding. J. Clin. Invest. 110, 515-526 (2002).

84. Cui, X., Rouhani, F.N., Hawari, F. \& Levine, S.J. An aminopeptidase, ARTS-1, is required for interleukin-6 receptor shedding. J. Biol. Chem. 278, 28677-28685 (2003).

85. Cui, X., Rouhani, F.N., Hawari, F. \& Levine, S.J. Shedding of the type II IL-1 decoy receptor requires a multifunctional aminopeptidase, aminopeptidase regulator of TNF receptor type 1 shedding. J. Immunol. 171, 6814-6819 (2003). 